The Astrophysical Journal, 209:102-107, 1976 October 1

(C) 1976. The American Astronomical Society. All rights reserved. Printed in U.S.A.

\title{
ON THE ORIGIN OF THE 10 MICRON DEPRESSIONS IN THE SPECTRA OF COMPACT INFRARED SOURCES
}

\author{
JOHN KWAN \\ State University of New York, Stony Brook \\ AND \\ Nick Scoville \\ University of Massachusetts, Amherst \\ Received 1975 December 29; revised 1976 March 12
}

\begin{abstract}
The $10 \mu$ depression observed in the spectrum of a compact infrared object is usually ascribed to absorption by intervening cold silicate grains, and the underlying source spectrum is taken to be either a blackbody or a blackbody with superposed excess $10 \mu$ emission. We question this assumption of the underlying source spectrum for optically thick compact sources. We find, upon modeling both the objects BN and W3 IRS5, that the source actually emits less at the $10 \mu$ resonance than outside the resonance, so that a depression at $10 \mu$ already exists in the source spectrum. This difference in emission arises because, due to the higher opacity in the resonance, the observed $10 \mu$ radiation is produced further out in the source than is the radiation just outside the resonance. And the lower dust temperature further out gives rise to a weaker emission at $10 \mu$ than in the continuum. An observed $10 \mu$ depression can be largely due to this effect, and little or no intervening extinction is required.

This explanation of the $10 \mu$ depression leads to a correlation such that the magnitude of depression will increase with decreasing color temperature of the source. It also predicts no depression at $20 \mu$ for sources with color temperatures greater than $200 \mathrm{~K}$. Observations at $20 \mu$ would then be able to decide on the validity of this explanation.
\end{abstract}

Subject headings: infrared: sources - opacities

\section{INTRODUCTION}

Many compact infrared sources, notably the $\mathrm{BN}$ object in Orion and W3 IRS5, show a broad depression at $10 \mu$ (Gillett and Forrest 1973; Aitken and Jones 1973; Merrill and Soifer 1974) which is ascribed to the effect of a silicate resonance in the dust grains (Woolf and Ney 1969; Knacke and Thomson 1973). The depression is generally visualized as due to absorption by intervening cold dust. The background source spectrum near $10 \mu$ is taken to be either a blackbody or an emission spectrum similar to that observed at the Trapezium region (Aitken and Jones 1973; Merrill and Soifer 1974). In this model the optical depth at the silicate feature is easily obtained, and it is hoped that this opacity will then be a good indicator of visual extinction to the source. Implicit in this procedure, however, is the assumption that the observed $10 \mu$ depression is all due to foreground absorption. In this paper we point out that this assumption may not be correct for optically thick compact sources. We find, upon modeling both $\mathrm{BN}$ and W3 IRS5, that a large portion of the $10 \mu$ depression can be the result of a much weaker emission from the source at the $10 \mu$ resonance than in the neighboring continuum. The outer layers of the compact source can play a role analogous to that played by the atmosphere of a star in the formation of stellar absorption lines. In this explanation the $10 \mu$ depression is intrinsic to the source. Little or no foreground absorption by cold grains is called for. The observed spectrum is the source spectrum, and the source luminosity is equal to that directly obtained from integration of the observed energy distribution. For these optically thick compact objects, then, caution must be taken in interpreting and using the $10 \mu$ depressions as measures of optical extinction to the sources.

To justify the above contention, we first argue that the observed flux from a luminous source, such as BN or W3 IRS5, is unlikely to be the attenuated radiation from a star (Becklin, Neugebauer, and Wynn-Williams 1973; Merrill and Soifer 1974). For typical stars the stellar emission longward of $10 \mu$ is on the RayleighJeans portion of the blackbody curve, and the integrated flux longward of $10 \mu$ is a very small fraction of the total stellar luminosity. Since an observed compact infrared object usually peaks near 10 and $20 \mu$, if it is an attenuated star the actual stellar luminosity must be many times greater than what is directly obtained from integration of the observed energy distribution. As an example, if W3 IRS5, assumed to be at a distance of $3 \mathrm{kpc}$, were a star attenuated by $1 \mathrm{mag}$ at $20 \mu$ (in order that the resulting spectrum 
peaks at this wavelength), the actual stellar luminosity would be related to its effective temperature by

$$
L_{*}=6.10^{7}\left(T_{*} / 5.10^{3} \mathrm{~K}\right)^{3} L_{\odot} .
$$

Such a high luminosity is not only incompatible with the total observed infrared luminosity at the whole W3 region, but also with presently known stellar luminosities.

A more plausible explanation for the infrared radiation is thermal emission from hot grains (Becklin, Neugebauer, and Wynn-Williams 1973; Merrill and Soifer 1974). In this case one can estimate the temperature of the emitting grains from the color temperature of the observed spectrum, and the size of the dust cloud from the equivalent radius of a blackbody of the same color temperature and observed luminosity. These hot grains are heated by absorbing photons of higher energy than those they emit, and the ultimate energy supply must, of course, be derived from either a star or protostar. The luminosity of the latter dictates the luminosity of the radiation that finally escapes from the dust cloud. The spectrum of this emergent radiation, however, will be that of the radiatively heated dust, and will depend most crucially on the dust density distribution and the dust emissive properties. Because the emissivity at the $10 \mu$ silicate resonance is greatly different from that outside the resonance, the emergent flux at this resonance will in general be different from that outside the resonance. For an optically thick cloud, we see into different regions of the cloud at different wavelengths, depending on the opacity at each wavelength. The higher opacity at $10 \mu$ means that the observed emission at this wavelength will arise in a region of the dust cloud further out than where the emission just outside the resonance arises. In general, the dust temperature decreases with increasing radius because the energy is supplied from within. Then whether the emission at $10 \mu$ is more or less than the emission outside the resonance depends on the balance between the advantages for emission of a larger surface area and a higher emissivity, on the one hand, against the disadvantage of a cooler temperature on the other. Accordingly, in this paper we calculate the transfer of infrared radiation in an optically thick cloud, and study whether the emergent radiation at each of the $10 \mu$ and $20 \mu$ resonances is more or less than the continuum radiation.

Our discussion applies to those compact infrared sources that are optically thick, such as BN, W3 IRS5, AFCRL 809-2992, and RCW57 IRS1 (Frogel and Persson 1974). As examples, the sources BN and W3 IRS5 will be modeled. In the next section the numerical calculations are described. The results are presented in $\S$ III, followed by a discussion of the validity of the model and future experimental tests in $\S \mathrm{IV}$.

\section{MODEL}

The compact sources BN and W3 IRS5 are unresolved at $2^{\prime \prime}$. Each has an overall energy distribution that can be fitted approximately by a blackbody curve. We label the color temperature derived from this blackbody fit $T_{c}$, and the luminosity from integration of the observed energy distribution $L$. To help describe the numerical model, we define a parameter $r_{c}$ as the radius of a blackbody of the temperature $T_{c}$ and luminosity $L$, namely,

$$
r_{c}=\left(\frac{L}{4 \pi \sigma T_{c}^{4}}\right)^{1 / 2} .
$$

This characteristic radius is informative because it indicates the size of the region from which the continuum radiation is formed. Also, the continuum opacity at the frequency of peak emission is expected to be about unity into this radius.

The main purpose of this paper is to demonstrate that, for an optically thick cloud, there can be less emergent radiation at the $10 \mu$ resonance than outside the resonance. In illustrating this physical effect with numerical calculations, we aim more to determine the circumstances under which such a $10 \mu$ depression in the emergent spectrum prevails, and less to reproduce precisely an observed energy distribution and magnitude of depression. Thus, rather than having an elaborate parametrization of the dust cloud, we shall use only simple forms for the dust emissivity law and density distribution. For the emissivity law we utilize results from laboratory measurements of lunar grains by Knacke and Thomson (1973). For $\lambda>30 \mu, \mathrm{a} \lambda^{-2}$ dependence is taken, and for $\lambda<5 \mu$, a $\lambda^{-1}$ dependence. For simplicity we make the approximation of a very narrow resonance at $10 \mu$ and thus neglect its effect on the radiative equilibrium of the grains. This approximation simplifies the parametrization of the emissivity law, and also illustrates more clearly the dependence of the emergent flux on the emissivity at each wavelength. The ratio of the emissivity at $10 \mu$ to the continuum emissivity just outside the resonance is denoted by $\beta_{10 \mu}$. In the measurements by Knacke and Thomson, this ratio is about 6 at $10 \mu$ and decreases to unity at 8 and $13 \mu$. To simulate this variation within our approximation, we calculate the $10 \mu$ emission for $\beta_{10 \mu}=6,4$, and 2. A narrow resonance is also assumed at $20 \mu$. The corresponding ratio $\beta_{20 \mu}$ is taken to be 2 . Altogether then, the emissivity law used is

$$
\begin{array}{crl}
\epsilon(\nu)=\epsilon_{0}\left(\frac{30 \mu}{\lambda}\right)^{2} & \frac{c}{\nu}=\lambda \geq 30 \mu \\
=\epsilon_{0} & 5 \mu \leq \lambda \leq 30 \mu, \\
=\beta_{20 \mu} \epsilon_{0} & \text { but } \lambda \neq 20 \mu, \quad \neq 10 \mu \\
=\beta_{10 \mu} \epsilon_{0} & \lambda=20 \mu \\
=\epsilon_{0}\left(\frac{5 \mu}{\lambda}\right) & \lambda=10 \mu \\
=1 & \lambda \leq 1 \mu \leq 0.1 \mu .
\end{array}
$$


For the density distribution, a power-law form is assumed:

$$
n_{d}(r)=n_{d}\left(r_{c}\right)\left(\frac{r_{c}}{r}\right)^{\alpha} \quad \alpha=1 \quad \text { or } 2 .
$$

The dust density always appears together with the dust cross section $\sigma_{d}$ in a product, so that the significant quantity to specify is $n_{d}\left(r_{c}\right) \sigma_{d}$. It is, however, more informative to specify an associated quantity, the opacity into the radius $r_{c}$. At $5 \mu$, this opacity is

$$
\begin{aligned}
\tau_{5 \mu}\left(r_{c}\right) & =\int_{r_{c}}^{R} n_{d}(r) \sigma_{d} \epsilon_{0} d r \\
& =n_{d}\left(r_{c}\right) \sigma_{d} \epsilon_{0} r_{c} \quad \ln \frac{R}{r_{c}}, \quad \alpha=1 \\
& =n_{d}\left(r_{c}\right) \sigma_{d} \epsilon_{0} r_{c}\left(1-\frac{r_{c}}{R}\right), \quad \alpha=2,
\end{aligned}
$$

where $R$ is the outer radius of the cloud.. For the $r^{-2}$ density distribution, this outer radius is unimportant and the cloud can effectively extend to infinity. For the $r^{-1}$ density distribution, however, it is necessary to terminate the distribution at $R \gg r_{\mathrm{c}}$; otherwise it would be impossible to observe into the cloud. For this reason, and also for computational purposes, the cloud is truncated at $R=20 r_{c}$. There is negligible continuum and $10 \mu$ emission from $r \sim R$, so that this positioning of a cloud boundary in no way affects the distribution of the emergent radiation. Once the parameter $\tau_{5 \mu}\left(r_{c}\right)$ is chosen and a certain luminosity $L$ specified, the energy distribution of the emergent radiation is completely determined.

For computational purposes the dust cloud is also truncated at an inner radius of $0.1 r_{c}$. For example, in modeling BN, the dust envelope extends from an inner radius of $3.10^{13} \mathrm{~cm}$ to an outer radius of $6.10^{15} \mathrm{~cm}$. A star of luminosity $L$ and radius much smaller than $0.1 r_{c}$ is placed at the center. This is purely a computational trick to ensure that the luminosity of the radiation that finally emerges from the cloud equals $L$. The stellar photons are absorbed at the inner cloud boundary, and the dust temperature there will depend on the assumed stellar energy distribution and will be inaccurate, but this error would not affect the emergent energy distribution from the cloud, as the latter arises from $r \sim r_{c} \gg 0.1 r_{c}$, and the transfer of the infrared radiation from $0.1 r_{c}$ to $r_{c}$ quickly loses memory of the stellar temperature due to the low onefifth order dependence of the dust temperature upon the wavelength of the radiation incident on the grain (Scoville and Kwan 1976). In numerically calculating the dust temperature distribution, the cloud is divided into some 20 concentric shells between the inner and outer boundary, and the dust temperature in each shell is determined from radiative equilibrium. The pertinent equations governing the heating and cooling of a dust grain and the numerical methods of computation are described fully in Scoville and Kwan (1976).

We have attempted to model both the objects BN and W3 IRS5, with luminosities of $1.5 \cdot 10^{3} L_{\odot}$ and $3.5 \cdot 10^{4} L_{\odot}$, respectively. In each calculation, the parameter $\tau_{5 \mu}\left(r_{c}\right)$ is varied to obtain a continuum energy distribution between $1 \mu$ and $20 \mu$ that approximates the observed one. The emission at $10 \mu$ and $20 \mu$ is then calculated for the various values of $\beta_{10 \mu}$ and $\beta_{20 \mu}$.

\section{RESULTS}

The results of the numerical calculations are presented in Figures 1 and 2, which show the calculated energy distributions for BN and W3 IRS5, respectively. The solid curve refers to the calculated spectrum for an $r^{-1}$ density distribution, and the dashed curve for an $r^{-2}$ density distribution. In each case the emission arises from within a 1 " diameter aperture. The observed data, from a $5^{\prime \prime}$ beam, are taken from Becklin, Neugebauer, and Wynn-Williams (1973) for BN, and from Wynn-Williams, Becklin, and Neugebauer (1972) for W3 IRS5. The flux density at $10 \mu$ is not shown in the figures because it depends on $\beta_{10 \mu}$. It is given in Table 1A, where the ratio of this flux density to the continuum flux density just outside the resonance is tabulated for $\beta_{10 \mu}=2,4$, and 6 . The observed ratio at $10 \mu$ is $\sim 0.25$ at BN (Gillett and Forrest 1973), and $\leq 0.03$ at W3 IRS5 (Aitken and Jones 1973).

In all the cases calculated, there is less emission at $10 \mu$ than there is outside the resonance. This result, together with the approximate fit of the calculated energy distributions to the observed ones, lends

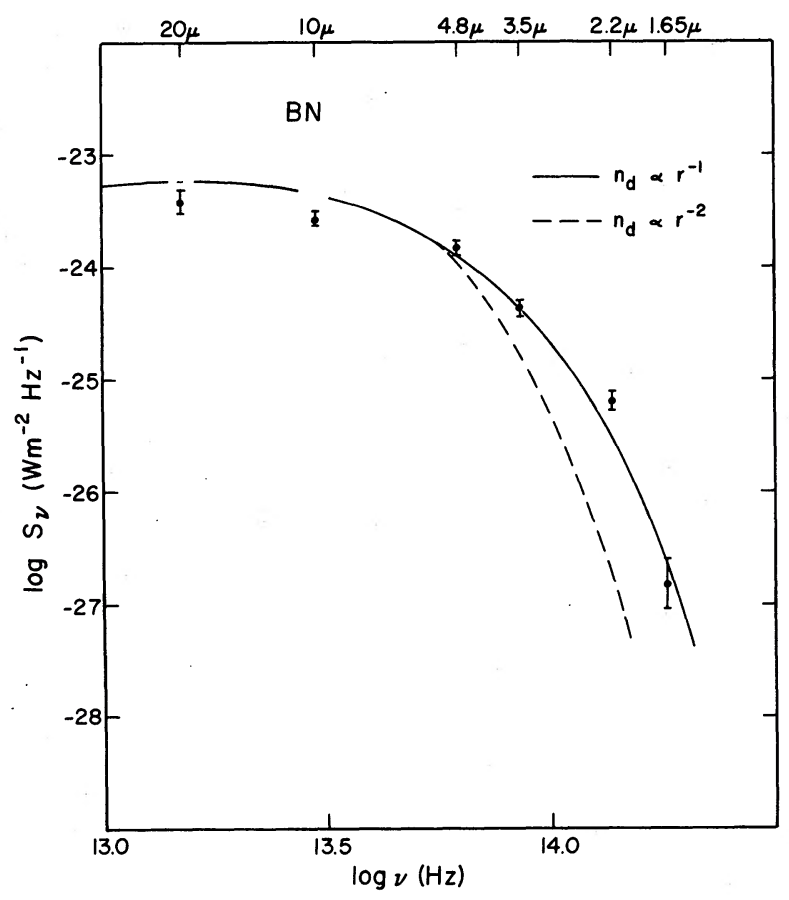

FIG. 1.-Continuum energy distributions of BN, calculated for both a $r^{-1}$ (solid curve) and $r^{-2}$ (dashed curve) density distribution. Observed data points are from Becklin, Neugebauer, and Wynn-Williams (1973). The emitted fluxes at $10 \mu$ and $20 \mu$ depend on the emissivities at the two silicate resonances and are given in Tables $1 \mathrm{~A}$ and $1 \mathrm{~B}$, respectively. 


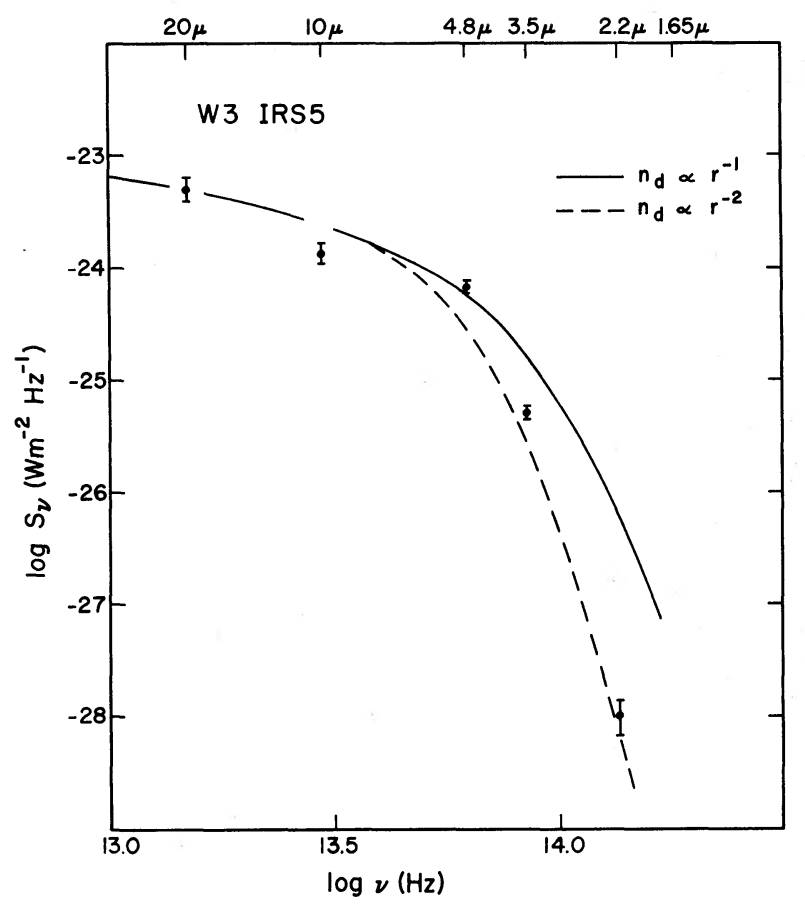

FIG. 2.-Calculated continuum energy distributions of W3 IRS5. Observed data points are from Wynn-Williams, Becklin, and Neugebauer (1972). The emitted fluxes at $10 \mu$ and $20 \mu$ are given in Tables $1 \mathrm{~A}$ and $1 \mathrm{~B}$.

credence to the claim that part or all of the $10 \mu$ depression observed at a compact object is due to there being less emission from the source at the $10 \mu$ resonance than outside the resonance.

The numerical calculations show a greater deficiency in $10 \mu$ emission, relative to the continuum, from W3 IRS5 than from BN. This is due to the lower color temperature of W3 IRS5. Thus, at the region where the $10 \mu$ emission is formed, the dust temperature is lower in the case of W3 IRS5 than it is in the case of BN. At temperatures lower than $500 \mathrm{~K}$, the dust emission at $10 \mu$ is on the exponentially decreasing portion of the blackbody curve, and its magnitude decreases rapidly with decreasing temperature. For this reason, the $10 \mu$ emission, relative to the continuum, is weaker for W3 IRS5 than for BN. In general, then, we expect a correlation such that the $10 \mu$ depression will be deeper the lower the color temperature of the source. Conversely, for sources of high color temperatures $(>1500 \mathrm{~K})$, a greater emission at $10 \mu$ than at the continuum will be expected.

The emission at $10 \mu$ also depends strongly on the value of $\beta_{10 \mu}$, as indicated in Table 1A. It decreases as $\beta_{10 \mu}$ is increased. This is expected, as with a larger value of $\beta_{10 \mu}$, and hence a higher $10 \mu$ opacity, the emission will be formed in a region further out in the dust cloud, and the disadvantage to emission due to a cooler dust temperature will more than balance against the advantages of a larger region of formation and a higher emissivity. Comparing the calculated
TABLE 1A

Ratio of the 10 Micron Flux Density TO THE CONTINUUM FluX DenSITY

\begin{tabular}{|c|c|c|c|c|}
\hline BN & $\beta_{10 \mu}=2$ & $\beta_{10 \mu}=4$ & $\beta_{10 \mu}=6$ & $\begin{array}{c}\text { Observed } \\
\text { Ratio at } 10 \mu\end{array}$ \\
\hline $\begin{array}{l}n_{d} \alpha r^{-1} \ldots \\
n_{d} \alpha r^{-2} \ldots\end{array}$ & $\begin{array}{l}0.73 \\
0.78\end{array}$ & $\begin{array}{l}0.43 \\
0.49\end{array}$ & $\begin{array}{l}0.27 \\
0.34\end{array}$ & $\sim 0.25$ \\
\hline \multicolumn{5}{|l|}{ W3 IRS5 } \\
\hline $\begin{array}{l}n_{d} \alpha r^{-1} \\
n_{d} \alpha r^{-2}\end{array} \ldots$ & $\begin{array}{l}0.69 \\
0.58\end{array}$ & $\begin{array}{l}0.22 \\
0.24\end{array}$ & $\begin{array}{l}0.08 \\
0.12\end{array}$ & $\lesssim 0.03$ \\
\hline
\end{tabular}

TABLE 1B

Ratio of the 20 Micron Flux Density TO THE CONTINUUM FluX Density

\begin{tabular}{|c|c|}
\hline $\mathrm{BN}$ & $\beta_{20 \mu}=2$ \\
\hline 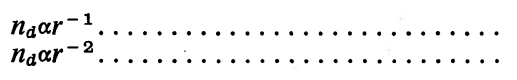 & $\begin{array}{l}1.06 \\
1.09\end{array}$ \\
\hline W3 IRS5 & \\
\hline $\begin{array}{l}n_{d} \alpha r^{-1} \ldots \ldots \cdots \\
n_{d} \alpha r^{-2} \ldots \ldots \cdots\end{array}$ & $\begin{array}{l}1.02 \\
0.99\end{array}$ \\
\hline
\end{tabular}

deficiencies in $10 \mu$ radiation with the observed values at BN and W3 IRS5, it is seen that, in order to explain the observations, $\beta_{10 \mu} \geqslant 6$ is required.

In general, the deficiency in $10 \mu$ emission is smaller for the $r^{-2}$ distribution than for the $r^{-1}$ distribution. This is because the falloff in density, and hence in opacity, with increasing radius is more rapid for the former distribution than for the latter. The $10 \mu$ emission will then arise from a region closer to where the continuum emission is formed in the case of the $r^{-2}$ density distribution, and, consequently, the difference between the emission at the resonance and the emission outside the resonance becomes smaller.

The $10 \mu$ emission is sensitive to the parameter $\tau_{5 \mu}\left(r_{c}\right)$, which was varied in the calculations to achieve a good fit of the calculated continuum energy distribution to the observed one. For the two models of BN in Figure 1, this parameter equals 1 for both density distributions. For the models of W3 IRS5, it is 0.75 for the case of the $r^{-1}$ distribution, and 0.5 for the case of the $r^{-2}$ distribution. As an example to illustrate the dependence of the $10 \mu$ emission on this parameter, when it was changed to 0.5 in the case of the $r^{-1}$ distribution which models W3 IRS5, the $10 \mu$ emission increased by a factor of about 2 for $\beta_{10 \mu}=4$ and 6 . Thus, while a deficiency in $10 \mu$ emission is always found, its calculated magnitude can be uncertain by a factor of 2 .

At $20 \mu$, the calculations show that there is no depression for BN or W3 IRS5. Indeed, for BN, the emission at the resonance is slightly greater than that just outside the resonance. The primary reason for this result is that dust emits effectively at $20 \mu$ at temperatures $\geqslant 200 \mathrm{~K}$, so that, for BN and W3 IRS5 which have color temperatures $\geqslant 300 \mathrm{~K}$, even though 
the $20 \mu$ radiation is formed in a region where the dust temperature is lower than that at the region where the continuum radiation is formed, this disadvantage to emission is not so severe that it overshadows the advantages of a larger region of formation and a higher emissivity. Only for sources of very low color temperatures $(<200 \mathrm{~K})$ would an intrinsic depression at $20 \mu$ be present.

\section{DISCUSSION}

In this section we discuss the validity of the model and compare the theoretical predictions with observations. Future experimental tests of this model are also proposed.

The unknown parameters in the model are the density distribution and the ratio $\beta$ of the emissivity at the silicate resonance to the continuum emissivity. In modeling $\mathrm{BN}$ and W3 IRS5, we have demonstrated that, assuming either a $r^{-1}$ or $r^{-2}$ density distribution, there is less emission at the $10 \mu$ resonance than outside the resonance, consequently leading to a depression at $10 \mu$ in the emergent spectrum. While the actual density distribution of neither object is known, we believe that, unless the density increases much more rapidly than $r^{-2}$ near the characteristic radius $r_{c}$, the general conclusion that a depression at $10 \mu$ is already present in the source spectrum will be correct. The other uncertain parameter, $\beta_{10 \mu}$, can actually be estimated in two different ways. First, the laboratory measurements of lunar grains by Knacke and Thomson (1973) give a peak value of 6. Second, observed spectra at cool stars, such as $O$ Ceti and $\mu$ Cephei (Woolf and Ney 1969), and at the Trapezium region (Stein and Gillett 1969) show an excess emission at $10 \mu$, and they can also aid in estimating the above ratio. The peak excess emission is about a factor of 5 greater than the continuum emission. If this result is ascribed to the effect of a higher emissivity at the feature and optically thin emission, then the ratio $\beta_{10 \mu}$ can be estimated from $\beta_{10 \mu} \exp \left(-\beta_{10 \mu} \tau\right) /$ $\exp (-\tau)=5$, where $\tau$ is the continuum opacity at $10 \mu$. As an example, for $\tau=0.03, \beta_{10 \mu}=6$. We believe, then, that a value of 6 for the peak ratio is not unreasonable.

The $10 \mu$ depression has been observed in the spectra of many compact infrared sources. The continuum energy distributions of these objects can, in general, be grouped into two types, (1) a power-law spectrum shortward of $20 \mu$, and (2), a blackbody spectrum. Those sources with a power-law spectrum, e.g., W51 IRS1 and W49 OH(2) (Gillett et al. 1975), are extended. Their continuum emission near $10 \mu$ is very optically thin, as deduced by comparing the brightness temperature with the color temperature (Wynn-Williams, Becklin, and Neugebauer 1972; Gillett et al. 1975). For these optically thin sources we expect the $10 \mu$ silicate feature to be in emission, similar to that observed at the Trapezium region. Any observed $10 \mu$ depression is then a result of foreground extinction. Gillett et al. (1975), in determining the $10 \mu$ opacity of the obscuring material, do find that an underlying source spectrum similar to the Trapezium emission rather than to a blackbody consistently gives a better fit to the observed feature.

Those compact infrared sources with a blackbody spectrum, e.g., BN and W3 IRS5, are unresolved at $2^{\prime \prime}$. They appear to be optically thick. For these sources, a substantial depression at $10 \mu$ can already exist in the source spectrum, as numerical models of BN and W3 IRS5 demonstrate. The observed $10 \mu$ depressions at these sources then may not be a true measure of foreground extinction. Among these objects the $10 \mu$ depressions also tend to increase in magnitude with lower color temperatures. Thus, in order of decreasing color temperature, we have $\mathrm{BN}$, AFCRL 809-2992, W3 IRS5, galactic center, and KL. For KL we expect an extremely deep depression. Gillett and Forrest (1973) found a much stronger depression at KL than at BN, while Aitken, Jones, and Penman (1974) found almost equal depressions. We believe the problem in separating $\mathrm{BN}$ from $\mathrm{KL}$, and the presence of several compact sources (Rieke, Low, and Kleinmann 1973) and a nearby H II region at $\mathrm{KL}$ make determining the $10 \mu$ feature associated with KL most uncertain. A similar difficulty may also be present for the galactic center source. If we consider the first three sources alone, the $10 \mu$ depression indeed increases from $\mathrm{BN}$ to W3 IRS5. Comparing with the actual magnitudes of depression, however, the theoretical calculations indicate that for W3 IRS5, the amount of depression predicted (10 $\mu$ flux density/ continuum flux density $\approx 0.1$ ) cannot account for the total observed depression (10 $\mu$ flux density/continuum flux density $\approx 0.03$ ). This may indicate that a higher value of $\beta_{10 \mu}, \sim 8$, is required or that some additional intervening absorption is necessary.

The model we have presented for the $10 \mu$ depression can also account for the $\mathrm{H}_{2} \mathrm{O}$ ice depression at $3.1 \mu$ (Gillett and Forrest 1973; Merrill and Soifer 1974). Assuming that $\mathrm{H}_{2} \mathrm{O}$ ice is present only in grains at temperatures $<270 \mathrm{~K}$, we find that, for the model of BN with an $r^{-1}$ density distribution, a value of 2.5 for the ratio of the emissivity at the $\mathrm{H}_{2} \mathrm{O}$ ice feature to the continuum emissivity is required to explain the observed depression.

Dyck and Beichman (1974) and Capps and Knacke (1975) have observed a correlation between the amount of polarization and the $10 \mu$ feature at the KleinmannLow nebula and the galactic center. Based on the Davis-Greenstein alignment mechanism, Dyck and Beichman found that the amount of polarization at $\mathrm{KL}$ can be produced, at a gas density of $3 \times 10^{4}$ $\mathrm{cm}^{-3}$ and temperature of $70 \mathrm{~K}$, with a 7 milligauss magnetic field and dust at $\sim 5 \mathrm{~K}$. In the present model, where the $10 \mu$ depression is intrinsic to the source spectrum, the grains are at a temperature not much lower than the observed color temperature; and an alignment mechanism that operates at high dust temperatures $(\geqslant 200 \mathrm{~K})$ is required, or this intrinsic model can account for at best a small portion of an observed depression.

A strong test of whether the $10 \mu$ depression arises because of intervening absorption (extrinsic) or 
because there is actually less emission from the source at the $10 \mu$ resonance than outside the resonance (intrinsic), is provided by observations at $20 \mu$. In the extrinsic case, even though the resonance at $20 \mu$ is weaker than that at $10 \mu$ by a factor of 2 or 3 , absorption at $20 \mu$ should be observable and consistent with the $10 \mu$ absorption; whereas, if the $10 \mu$ depression is intrinsic, the calculations (cf. Table 1B) show that there is no depression at $20 \mu$ for sources with color temperatures $>200 \mathrm{~K}$. Another test of the origin of the $10 \mu$ depression is provided by determining the size of the emitting region at each wave- length. In the intrinsic model the $10 \mu$ emission arises from a region further out in the dust cloud than where the continuum emission just outside the resonance arises. Then, by measuring the sizes of a compact object at $8 \mu, 10 \mu$, and $13 \mu$, it should be found, if the intrinsic model were correct, that the source size at $10 \mu$ was bigger than that at $8 \mu$ or $13 \mu$. However, since these sources are expected to have sizes $<1^{\prime \prime}$ at $10 \mu$, the effect may be difficult to observe.

We thank Dr. R. Knacke and an unknown referee for helpful comments.

\section{REFERENCES}

Aitken, D. K., and Jones, B. 1973, Ap. J., 184, 127.

Aitken, D. K., Jones, B., and Penman, J. 1974, Proceedings of the Eighth ESLAB Symposium, ed. A. F. M. Morwood (ESRO SP-105), p. 43.

Becklin, E. E., Neugebauer, G., and Wynn-Williams, C. G. 1973, Ap. J. (Letters), 182, L17.

Capps, R., and Knacke, R. 1976, preprint.

Dyck, H. M., and Beichman, C. A. 1974, Ap. J., 194, 57

Frogel, J. A., and Persson, S. E. 1974, Ap. J., 192, 351.

Gillett, F. C., and Forrest, W. J. 1973, Ap. J., 179, 483

Gillett, F. C., Forrest, W. J., Merrill, K. M., Capps, R. W., and Soifer, B. T. 1975, Ap. J., 200, 609

Knacke, R. F., and Thomson, R. K. 1973, Pub. A.S.P., 85, 341.

JoHN KwAN: Bell Laboratories, Murray Hill, NJ 07974

N. Z. Scoville: Astronomy Department, University of Massachusetts, Amherst, MA 01002
Merrill, K. M., and Soifer, B. T. 1974, Ap. J. (Letters), 189, L27.

Rieke, G. H., Low, F. J., and Kleinmann, D. E. 1973, Ap. J. (Letters), 186, L17.

Scoville, N. Z., and Kwan, J. 1976, Ap. J., 206, 718.

Stein, W. A., and Gillett, F. C. 1969, Ap. J. (Letters), 155, L197.

Woolf, N. J., and Ney, E. P. 1969, Ap. J. (Letters), 155, L181.

Wynn-Williams, C. G., Becklin, E. E., and Neugebauer, G. 1972, M.N.R.A.S., 160, 1. 